\title{
Approximated Fractional Order Chebyshev Lowpass Filters
}

\author{
Todd Freeborn, ${ }^{1}$ Brent Maundy, ${ }^{2}$ and Ahmed S. Elwakil ${ }^{3}$ \\ ${ }^{1}$ Tangent Design Engineering Ltd., 27197 Avenue Northeast, Calgary, AB, Canada T2A 2L9 \\ ${ }^{2}$ Department of Electrical and Computer Engineering, University of Calgary, 2500 University Dr. N. W., Calgary, \\ AB, Canada T2N $1 N 4$ \\ ${ }^{3}$ Department of Electrical and Computer Engineering, University of Sharjah, P.O. Box 27272, UAE
}

Correspondence should be addressed to Todd Freeborn; todd.freeborn@gmail.com

Received 13 June 2014; Accepted 21 August 2014

Academic Editor: Guido Maione

Copyright (C) 2015 Todd Freeborn et al. This is an open access article distributed under the Creative Commons Attribution License, which permits unrestricted use, distribution, and reproduction in any medium, provided the original work is properly cited.

We propose the use of nonlinear least squares optimization to approximate the passband ripple characteristics of traditional Chebyshev lowpass filters with fractional order steps in the stopband. MATLAB simulations of $(1+\alpha),(2+\alpha)$, and $(3+\alpha)$ order lowpass filters with fractional steps from $\alpha=0.1$ to $\alpha=0.9$ are given as examples. SPICE simulations of 1.2, 1.5, and 1.8 order lowpass filters using approximated fractional order capacitors in a Tow-Thomas biquad circuit validate the implementation of these filter circuits.

\section{Introduction}

Fractional calculus, the branch of mathematics concerning differentiations and integrations to noninteger order, has been steadily migrating from the theoretical realms of mathematicians into many applied and interdisciplinary branches of engineering [1]. From the import of these concepts into electronics for analog signal processing emerged the field of fractional order filter design. This import into filter design has yielded much recent progress in theory [2-6], noise analysis [7], stability analysis [8], implementation [9-13], and applications $[14,15]$. These filter circuits have all been designed using the fractional Laplacian operator, $s^{\alpha}$, because the algebraic design of transfer functions is much simpler than solving the difficult time domain representations of fractional derivatives. One definition of a fractional derivative of order $\alpha$ is given by the Caputo derivative [16] as

$$
{ }_{a}^{C} D_{t}^{\alpha} f(t)=\frac{1}{\Gamma(\alpha-n)} \int_{a}^{t} \frac{f^{(n)}(\tau) d \tau}{(t-\tau)^{\alpha+1-n}},
$$

where $\Gamma(\cdot)$ is the gamma function and $n-1 \leq \alpha \leq n$. The Caputo definition of a fractional derivative is often used over other approaches because the initial conditions for this definition take the same form as the more familiar integer order differential equations. Applying the Laplace transform to the fractional derivative of (1) with lower terminal $a=0$ yields

$$
\mathscr{L}\left\{{ }_{0}^{C} D_{t}^{\alpha} f(t)\right\}=s^{\alpha} F(s)-\sum_{k=0}^{n-1} s^{\alpha-k-1} f^{(k)}(0),
$$

where $s^{\alpha}$ is also referred to as the fractional Laplacian operator. With zero initial conditions (2) can be simplified to

$$
\mathscr{L}\left\{{ }_{0}^{C} D_{t}^{\alpha} f(t)\right\}=s^{\alpha} F(s) .
$$

Therefore it becomes possible to define a general fractance device with impedance proportional to $s^{\alpha}$ [17], where the traditional circuit elements are special cases of the general device when the order is $-1,0$, and 1 for a capacitor, resistor, and inductor, respectively. The expressions of the voltage across a traditional capacitor are defined by integer order integration of the current through it. This element can be expanded to the fractional domain using noninteger order integration which results in the time domain expression for the voltage across the fractional order capacitor given by

$$
v_{C}^{\alpha}(t)=\frac{1}{C \Gamma(\alpha)} \int_{0}^{t} \frac{i(\tau)}{(t-\tau)^{1-\alpha}} d \tau,
$$


where $0 \leq \alpha \leq 1$ is the fractional orders of the capacitor, $i(t)$ is the current through the element, $C$ is the capacitance with units $\mathrm{F} / \mathrm{s}^{1-\alpha}$, and (s) is a unit of time not to be mistaken with the Laplacian operator. Note that we will refer to the units of these devices as (F) for simplicity.

By applying the Laplace transform to (4) with zero initial conditions the impedance of this fractional order element is given as $Z_{C}^{\alpha}(s)=1 / s^{\alpha} C$. Using this element in electrical circuits increases the range of responses that can be realized, expanding them from the narrow integer subset to the more general fractional domain. While these devices are not yet commercially available, recent research regarding their manufacture and production shows very promising results [18-20]. Therefore, it is becoming increasingly important to develop the theory behind using these fractional elements so that when they are available their unique characteristics can be fully taken advantage of.

In traditional filter design, ideal filters are approximated using methods that include Butterworth, Chebyshev, Elliptic, and Bessel filters. These filters attempt to approximate the ideal frequency response given by

$$
H(\omega)= \begin{cases}1, & \omega<\omega_{c} \\ 0, & \text { elsewhere }\end{cases}
$$

for a lowpass filter that passes all frequencies below the cutoff frequency $\left(\omega_{c}\right)$ with no attenuation and removes all frequencies above. A necessary condition for physically realizable filters though is to satisfy the Paley-Wiener criterion [21] which requires a nonzero magnitude response. Hence, ideal filters are not physically realizable because they have a magnitude of zero in a certain frequency range. However, in [21] it was suggested that ideal filters when viewed from the fractional order perspective might not require satisfying the Paley-Wiener criterion to be physically realizable. If fractional order filters do not require satisfying the PaleyWiener criterion it marks another significant different over their integer order counterparts; which requires further investigation to determine conclusively.

In this work we use a nonlinear least squares optimization routine to determine the coefficients of a fractional order transfer function required to approximate the passband ripple characteristics of traditional Chebyshev lowpass filters. MATLAB simulations of $(1+\alpha),(2+\alpha)$, and $(3+\alpha)$ order lowpass filters with fractional steps from $\alpha=0.1$ to $\alpha=0.9$ designed using this process are given as examples. SPICE simulations of 1.2, 1.5, and 1.8 order lowpass filters using approximated fractional order capacitors in a Tow-Thomas biquad circuit validate the implementation of these filter circuits.

1.1. Approximated Chebyshev Response. Fractional order lowpass filters with order $(1+\alpha)$ have previously been designed in $[9,22]$ using the transfer function given by

$$
H_{\mathrm{LP}}^{1+\alpha}(s)=\frac{a_{0}}{a_{1} s^{1+\alpha}+a_{2} s^{\alpha}+1}
$$

and realized using various topologies including a TowThomas biquad [9], fractional $\mathrm{RL}_{\beta} \mathrm{C}_{\alpha}$ circuits, and field programmable analog arrays (FPAAs) [22]. In [22] the coefficients of (6) were selected to approximate the flat passband response of the Butterworth filters. These coefficients were selected using a numerical search that compared the passband of the fractional filter to the Butterworth approximation over the frequency range $\omega=0.01 \mathrm{rad} / \mathrm{s}$ to $1 \mathrm{rad} / \mathrm{s}$ and returned the coefficients that yielded the lowest error over this region.

A similar method can be applied to determine the coefficients of (6) required to approximate the ripple characteristics in the passband of the Chebyshev approximation. Here a nonlinear least squares fitting is used that attempts to solve the problem

$$
\begin{aligned}
\min _{x}\left\||H(x, \omega)|-\left|C_{n}(\omega)\right|\right\|_{2}^{2} \\
=\min _{x} \sum_{i=1}^{k}\left(\left|H\left(x, \omega_{i}\right)\right|-\left|C_{n}\left(\omega_{i}\right)\right|\right)^{2}
\end{aligned}
$$

$$
\text { s.t. } x>0.1 \text {, }
$$

where $x$ is the vector of filter coefficients, $|H(x)|$ is the magnitude response using (6) calculated using $x,\left|C_{n}(j \omega)\right|$ is the normalized $n$th order Chebyshev magnitude response, $\left|H\left(x, \omega_{i}\right)\right|$ and $\left|C_{n}\left(\omega_{i}\right)\right|$ are the magnitude responses of (6) and $n$th order Chebyshev approximation at frequency $\omega_{i}$, and $k$ is the total number of data points in the collected magnitude response. This routine aims to find the coefficients that minimize the error between the magnitude response of (6) and the Chebyshev approximation. The constraint $(x>$ 0.1 ) is added for this problem because negative coefficients are not physically possible and to return values will be easily realized in hardware. This is not the first application of optimization routines in the field of fractional filters. Previously, optimization routines have been employed in [23] to generate approximations of $1 /(s+1)^{\alpha}$ for simulation and further realization for audio applications.

Applying the nonlinear least squares fitting over the frequency range $\omega=1 \times 10^{-5} \mathrm{rad} / \mathrm{s}$ to $1 \mathrm{rad} / \mathrm{s}$ using (6) and the second order Chebyshev filter designed with a ripple of $3 \mathrm{~dB}$ with transfer function

$$
C_{2}(s)=\frac{0.5012}{s^{2}+0.6449 s+0.7079}
$$

yields the coefficients given in Table 1 for orders $\alpha=0.2$, 0.5 , and 0.8 . The $3 \mathrm{~dB}$ ripple was selected over smaller ripple magnitudes to highlight the difference in ripple size using the fractional order response over the integer order response. The coefficients were determined in MATLAB using the lsqcurvefit function to implement the NLSF described by (7). This function uses the trust-region-reflective algorithm [24] with termination tolerances of the function value and the solution set to $10^{-6}$.

The magnitude responses using these coefficients, as well as those determined for orders $\alpha=0.1$ to 0.9 in steps of 0.1, are given in Figure 1(a) as dashed lines. For comparison, the magnitude responses of first and second order Chebyshev lowpass filters with $3 \mathrm{~dB}$ ripples are also given. From these responses attenuations with fractional steps between the first 
TABLE 1: Coefficient values for $(1+\alpha),(2+\alpha)$, and $(3+\alpha)$ fractional order transfer functions to approximate Chebyshev passband response.

\begin{tabular}{|c|c|c|c|c|c|c|c|c|c|}
\hline Order & $\alpha$ & $a_{0}$ & $a_{1}$ & $a_{2}$ & $a_{3}$ & $a_{4}$ & $a_{5}$ & $a_{6}$ & $\left|\theta_{W}\right|_{\text {min }}$ \\
\hline \multirow{3}{*}{$1+\alpha$} & 0.2 & 0.7495 & 0.5095 & 0.1 & - & - & - & - & $16.5^{\circ}$ \\
\hline & 0.5 & 0.7135 & 1.215 & 0.1 & - & - & - & - & $13.1^{\circ}$ \\
\hline & 0.8 & 0.7107 & 1.5281 & 0.5092 & - & - & - & - & $11.9^{\circ}$ \\
\hline \multirow{3}{*}{$2+\alpha$} & 0.2 & 1.061 & 2.246 & 0.1 & 2.1 & 0.2577 & - & - & $12.2^{\circ}$ \\
\hline & 0.5 & 1.013 & 3.652 & 0.1 & 2.912 & 0.2608 & - & - & $10.5^{\circ}$ \\
\hline & 0.8 & 1.002 & 4.252 & 1.210 & 3.481 & 0.1 & - & - & $10.0^{\circ}$ \\
\hline \multirow{3}{*}{$3+\alpha$} & 0.2 & 0.7339 & 3.735 & 0.1 & 2.464 & 2.920 & 0.1 & 0.1 & $10.1^{\circ}$ \\
\hline & 0.5 & 0.7146 & 5.734 & 0.1 & 2.878 & 3.907 & 0.1 & 0.3466 & $9.6^{\circ}$ \\
\hline & 0.8 & 0.7087 & 6.256 & 1.246 & 6.592 & 0.1 & 1.894 & 0.1906 & $9.2^{\circ}$ \\
\hline
\end{tabular}

and second order Chebyshev responses, with $-20 \mathrm{~dB} /$ decade and $-40 \mathrm{~dB} /$ decade attenuations, respectively, are visible above frequencies of $10 \mathrm{rad} / \mathrm{s}$. In the inset highlighting the responses around $1 \mathrm{rad} / \mathrm{s}$ the increase in ripple size for increasing order is visible reaching values of $-2.5867 \mathrm{~dB}$, $-0.8854 \mathrm{~dB}$, and $0.0356 \mathrm{~dB}$ for $\alpha=0.2,0.5$, and 0.8 , respectively. Therefore, using this method filter responses of order $(1+\alpha)$ with both fractional-step attenuation in the stopband and fractional ripple characteristics can be created.

This method can also be applied to create higher order filters with fractional characteristics in both stopband and passband. The fractional transfer function for a $(2+\alpha)$ filter response, developed by combining (6) and a bilinear transfer function, is given below:

$$
H_{\mathrm{LP}}^{2+\alpha}(s)=\frac{a_{0}}{a_{1} s^{2+\alpha}+a_{2} s^{1+\alpha}+a_{3} s+a_{4} s^{\alpha}+1} .
$$

Applying (7) from $\omega=1 \times 10^{-5} \mathrm{rad} / \mathrm{s}$ to $1 \mathrm{rad} / \mathrm{s} \mathrm{using} \mathrm{(9)} \mathrm{and}$ the third order Chebyshev filter designed with a ripple of $3 \mathrm{~dB}$ given by the transfer function

$$
C_{3}(s)=\frac{0.2506}{s^{3}+0.5972 s^{2}+0.9283 s+0.2506}
$$

yields the parameters given in Table 1 for orders $\alpha=0.2,0.5$, and 0.8 . The magnitude responses using these parameters, as well as those determined for orders $\alpha=0.1$ to 0.9 in steps of 0.1, are given in Figure 1(b) as dashed lines. For comparison the magnitude responses of second and third order Chebyshev lowpass filters with $3 \mathrm{~dB}$ ripples are also given. Again, fractional steps between the integer order magnitude responses are visible above frequencies of $10 \mathrm{rad} / \mathrm{s}$. Similar to the $(1+\alpha)$ filter, the size of the ripples in the passband increases with the fractional order $(\alpha)$.

This method is further applied to create a $(3+\alpha)$ filter response, developed by combining (6) and a biquadratic transfer function, with transfer function given by

$$
\begin{aligned}
& H_{\mathrm{LP}}^{3+\alpha}(s) \\
& \quad=\frac{a_{0}}{a_{1} s^{3+\alpha}+a_{2} s^{2+\alpha}+a_{3} s^{2}+a_{4} s^{1+\alpha}+a_{5} s+a_{6} s^{\alpha}+1} .
\end{aligned}
$$

Applying (7) from $\omega=1 \times 10^{-5} \mathrm{rad} / \mathrm{s}$ to $1 \mathrm{rad} / \mathrm{s}$ using (11) and the fourth order Chebyshev filter designed with a ripple of $3 \mathrm{~dB}$ given by the transfer function

$$
C_{4}(s)=\frac{0.1253}{s^{4}+0.5816 s^{3}+1.1691 s^{2}+0.4048 s+0.1770}
$$

yields the parameters given in Table 1 for orders $\alpha=0.2,0.5$, and 0.8 . The magnitude responses using these parameters, as well as those determined for orders $\alpha=0.1$ to 0.9 in steps of 0.1 , are given in Figure 1(c) as dashed lines. For comparison the magnitude responses of third and fourth order Chebyshev lowpass filters with $3 \mathrm{~dB}$ ripples are given.

While these filters exhibit fractional characteristics in their magnitude response, in the next section we analyze their stability to ensure that these fractional transfer functions are physically realizable.

1.2. Stability. Analyzing the stability of fractional filters requires conversion of the $s$-domain transfer functions to the $W$-plane defined in [25]. This transforms the transfer function from fractional order to integer order to be analyzed using traditional integer order analysis methods. The process for this analysis can be done using the following steps.

(1) Convert the fractional transfer function to the $W$ plane using the transformations $s=W^{m}$ and $\alpha=k / m$ [25].

(2) Select $k$ and $m$ for the desired $\alpha$ value.

(3) Solve the transformed transfer function for all poles in the $\mathrm{W}$-plane and if any of the absolute pole angles, $\left|\theta_{W}\right|$, are less than $\pi / 2 \mathrm{~m} \mathrm{rad} / \mathrm{s}$ then the system is unstable; otherwise if all $\left|\theta_{W}\right|>\pi / 2 m$ then the system is stable.

Applying this process to the denominators of (6), (9), and (11) yields the characteristic equations in the $W$-plane given by

$$
\begin{gathered}
0=a_{1} W^{m+k}+a_{2} W^{k}+1, \\
0=a_{1} W^{2 m+k}+a_{2} W^{k+m}+a_{3} W^{m}+a_{4} W^{k}+1, \\
0=a_{1} W^{3 m+k}+a_{2} W^{2 m+k}+a_{3} W^{2 m}+a_{4} W^{m+k} \\
+a_{5} W^{k}+a_{6} W^{m}+1 .
\end{gathered}
$$




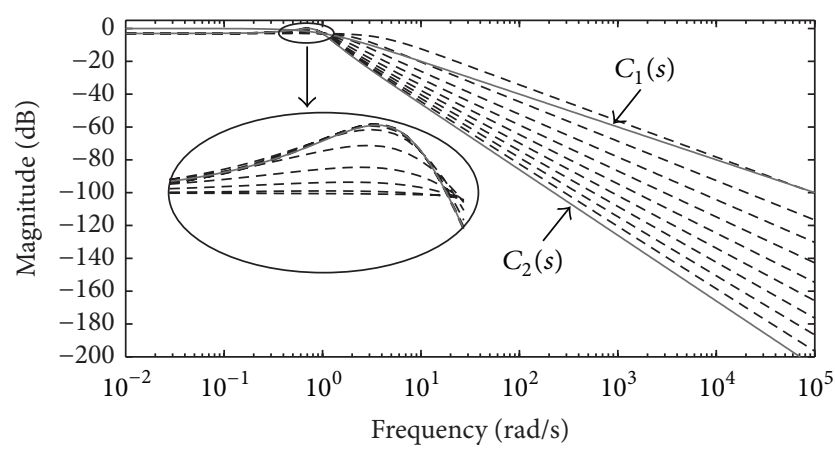

(a)

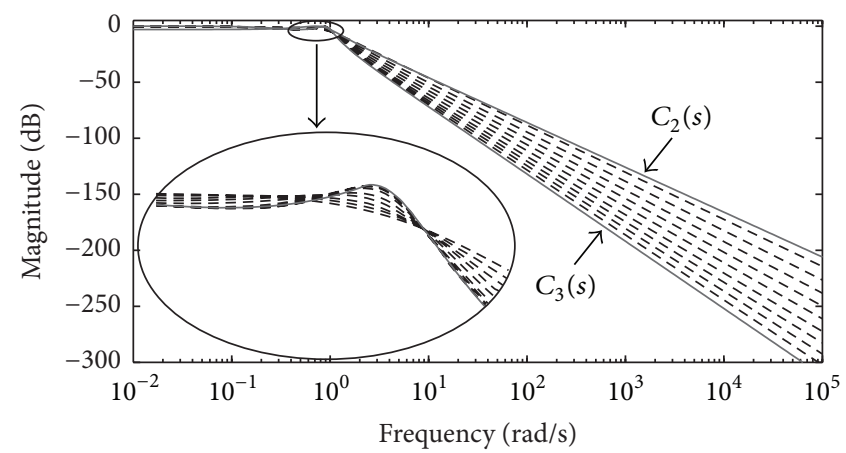

(b)

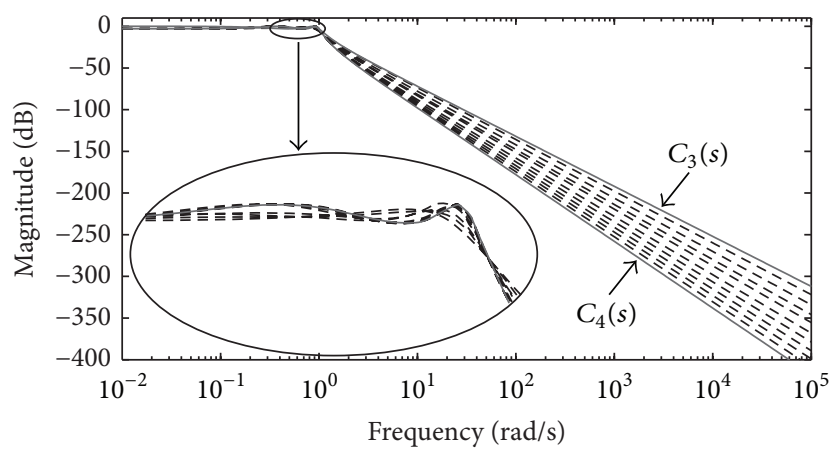

(c)

FIGURE 1: Simulated magnitude responses of (a) $(1+\alpha)$, (b) $(2+\alpha)$, and (c) $(3+\alpha)$ lowpass fractional order filter circuits for $\alpha=0.1$ to 0.9 in steps of 0.1 with coefficients selected to approximate Chebyshev passband response using nonlinear least squares fitting.

The roots of (13)-(15) for $\alpha=0.1,0.5$, and 0.9 were calculated with $k=1,5$, and 9 , respectively, when $m=10$. The minimum root angles, $\left|\theta_{W}\right|_{\text {min }}$, for each case are given in Table 1 . The angles for each case are greater than the minimum required angle, $\left|\theta_{W}\right|>\pi / 2 m=9^{\circ}$, confirming that each filter using the coefficients in Table 1 is stable and can be physically realized.

\section{Circuit Realization}

The fractional order transfer function (6) can be realized by the Tow-Thomas biquad, given in Figure 2, when $C_{2}$ is replaced with a fractional order capacitor with impedance $Z_{C_{2}}=1 / s^{\alpha} C_{2}$ and $0 \leq \alpha \leq 1$. This topology was previously employed in [9] to realize fractional order filter circuits with flat passband characteristics and fractional attenuations in the stopband. The transfer function of the fractional order TowThomas biquad at the noninverting lowpass output is given by

$$
\frac{V_{\mathrm{o}}(s)}{V_{\mathrm{in}}(s)}=\frac{R_{3} R_{5} / R_{4} R_{6}}{s^{1+\alpha} R_{2} R_{3} C_{1} C_{2}+s^{\alpha}\left(R_{2} R_{3} C_{2} / R_{1}\right)+1}
$$

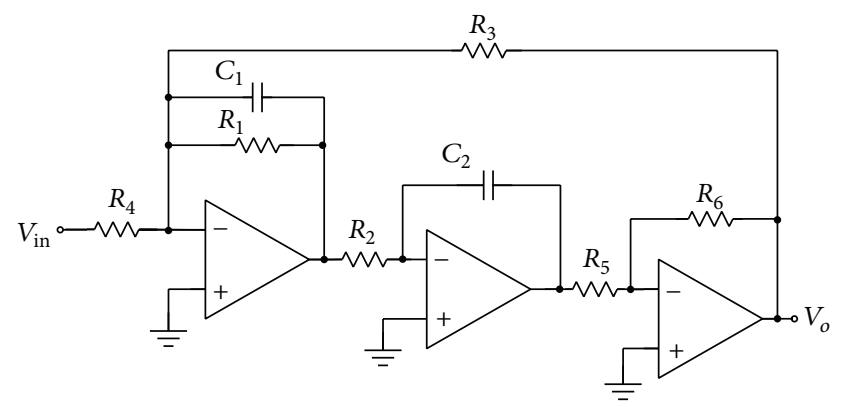

FIGURE 2: Tow-Thomas biquad topology.

Comparing the coefficients of (16) to (6) yields the following relationships:

$$
\begin{aligned}
& a_{0}=\frac{R_{3} R_{5}}{R_{4} R_{6}}, \\
& a_{1}=R_{2} R_{3} C_{1} C_{2}, \\
& a_{2}=\frac{R_{2} R_{3} C_{2}}{R_{1}} .
\end{aligned}
$$


TABLE 2: Component values to realize (16) when $\alpha=0.2,0.5$, and 0.8 .

\begin{tabular}{lccc}
\hline Component & \multicolumn{3}{c}{ Values for FLPF of order } \\
& 1.2 & 1.5 & 1.8 \\
\hline$C_{1}(\mathrm{~F})$ & & $0.159 \mu$ & \\
$C_{2}(\mathrm{~F})$ & $173.9 \mu$ & $12.6 \mu$ & $0.915 \mu$ \\
$R_{1}(\Omega)$ & 5095 & $12.147 \mathrm{k}$ & $3.001 \mathrm{k}$ \\
$R_{2}(\Omega)$ & 679.8 & 1702.5 & 2150.1 \\
$R_{3}(\Omega)$ & 749.5 & 713.5 & 710.7 \\
$R_{4}, R_{5}, R_{6}(\Omega)$ & & 1000 & \\
\hline
\end{tabular}

Using (17) to (19) we have 3 design equations and 8 variables yielding 5 degrees of freedom in our selection of the component values required to realize the desired $a_{0}, a_{1}$, and $a_{2}$ values for the approximated fractional Chebyshev magnitude response. Therefore, setting $C_{1}=C_{2}=1 \mathrm{~F}$ and $R_{4}=R_{5}=$ $R_{6}=1 \Omega$ the design equations for the remaining components become

$$
\begin{gathered}
a_{0}=R_{3}, \\
a_{1}=R_{2} R_{3}, \\
a_{2}=\frac{R_{2} R_{3}}{R_{1}} .
\end{gathered}
$$

Solving equations $(20)$ to $(22)$ with the $(1+\alpha)$ coefficients from Table 1 for $R_{1}, R_{2}$, and $R_{3}$ yields the component values in Table 2 to realize the approximated fractional Chebyshev magnitude response, magnitude scaled by a factor of 1000 and frequency shifted to $1 \mathrm{kHz}$.

2.1. SPICE Simulations. Although there has been much progress towards realizing fractional order capacitors [1820] there are currently no commercial devices using these processes available to implement these circuits, though their increasing progress towards commercialization highlights the need to research their use in electronic circuits to take advantage of their unique characteristics when they do become available. Until commercial devices with the desired characteristics become available integer order approximations must be used to realize fractional circuits. There are many methods to create an approximation of $s^{\alpha}$ which include continued fraction expansions (CFEs) as well as rational approximation methods [26]. These methods present a large array of approximations with the accuracy and approximated frequency band increasing as the order of the approximation increases. Here, a CFE method [27] was selected to model the fractional order capacitors for SPICE simulations. Collecting eight terms of the CFE yields a 4 th order approximation of the fractional capacitor that can be physically realized using the RC ladder network in Figure 3.

The component values required for the 4 th order approximation of the fractional capacitances with values of $173.9 \mu \mathrm{F}$, $12.6 \mu \mathrm{F}$, and $0.915 \mu \mathrm{F}$ and orders $0.2,0.5$, and 0.8 , respectively, using the RC ladder network in Figure 3, shifted to a center frequency of $1 \mathrm{kHz}$, are given in Table 3.
TABLE 3: Component values to realize 4th order approximations of fractional capacitors with values of $173.9 \mu \mathrm{F}, 12.6 \mu \mathrm{F}$, and $0.915 \mu \mathrm{F}$ and orders $0.2,0.5$, and 0.8 , respectively. The center frequency is $1 \mathrm{kHz}$.

\begin{tabular}{lccc}
\hline Component & \multicolumn{3}{c}{ Values } \\
& $\begin{array}{c}C_{2}=173.9 \mu \mathrm{F} \\
\alpha=0.2\end{array}$ & $\begin{array}{c}C_{2}=12.6 \mu \mathrm{F} \\
\alpha=0.5\end{array}$ & $\begin{array}{c}C_{2}=0.915 \mu \mathrm{F} \\
\alpha=0.8\end{array}$ \\
\hline$R_{a}(\Omega)$ & 431.8 & 111.2 & 18.4 \\
$R_{b}(\Omega)$ & 285.2 & 251.7 & 92.8 \\
$R_{c}(\Omega)$ & 241.4 & 378.7 & 236.2 \\
$R_{d}(\Omega)$ & 337.2 & 888.9 & 981.6 \\
$R_{e}(\Omega)$ & 1020.2 & 7369.7 & $53.1 \mathrm{k}$ \\
$C_{b}(\mathrm{~F})$ & $53.5 \mathrm{n}$ & $83.7 \mathrm{n}$ & $301.3 \mathrm{n}$ \\
$C_{c}(\mathrm{~F})$ & $375.5 \mathrm{n}$ & $295.6 \mathrm{n}$ & $585.2 \mathrm{n}$ \\
$C_{d}(\mathrm{~F})$ & $1.114 \mu$ & $536.5 \mathrm{n}$ & $635.3 \mathrm{n}$ \\
$C_{e}(\mathrm{~F})$ & $2.804 \mu$ & $693.7 \mathrm{n}$ & $273.0 \mathrm{n}$ \\
\hline
\end{tabular}

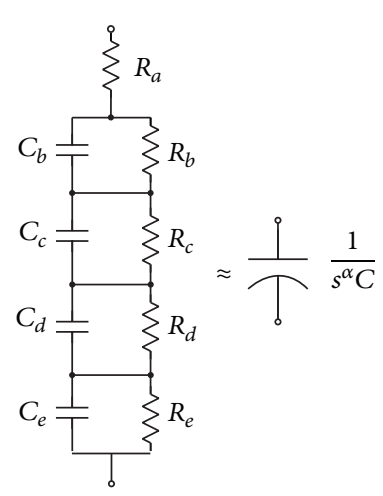

FIGURE 3: RC ladder structure to realize a 4 th order integer approximation of a fractional order capacitor.

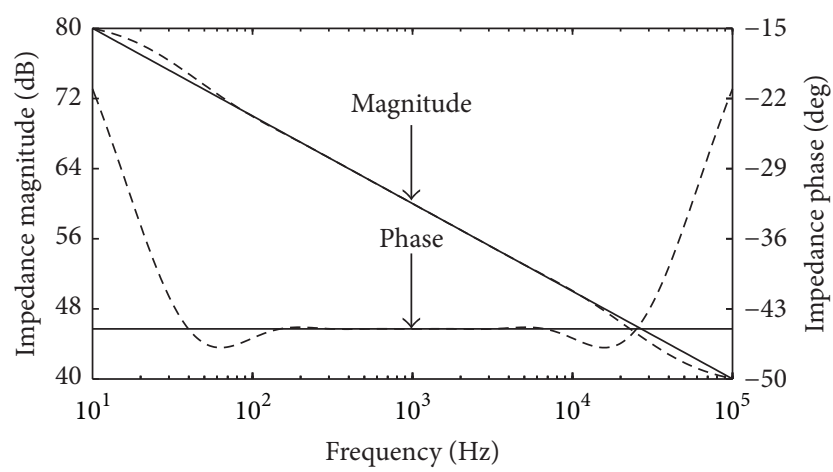

FIGURE 4: Magnitude and phase response of the approximated fractional order capacitor (dashed) compared to the ideal (solid) with capacitance of $12.6 \mu \mathrm{F}$ and order 0.5 after scaling to a center frequency of $1 \mathrm{kHz}$.

The magnitude and phase of the ideal (solid line) and 4th order approximated (dashed) fractional order capacitor with capacitance $12.6 \mu \mathrm{F}$ and order $\alpha=0.5$, shifted to a center frequency of $1 \mathrm{kHz}$, are presented in Figure 4. From this figure we observe that the approximation is very good over almost 4 decades, from $200 \mathrm{~Hz}$ to $70 \mathrm{kHz}$, for the magnitude and 


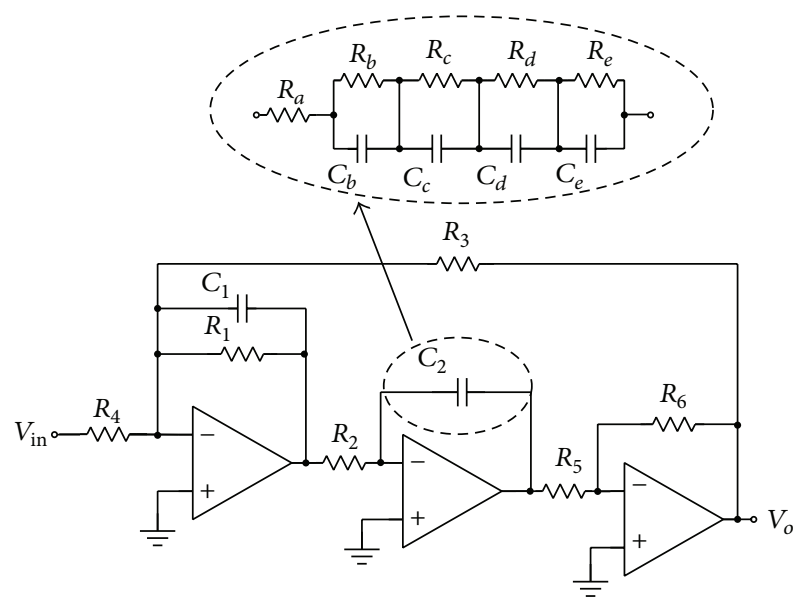

(a)

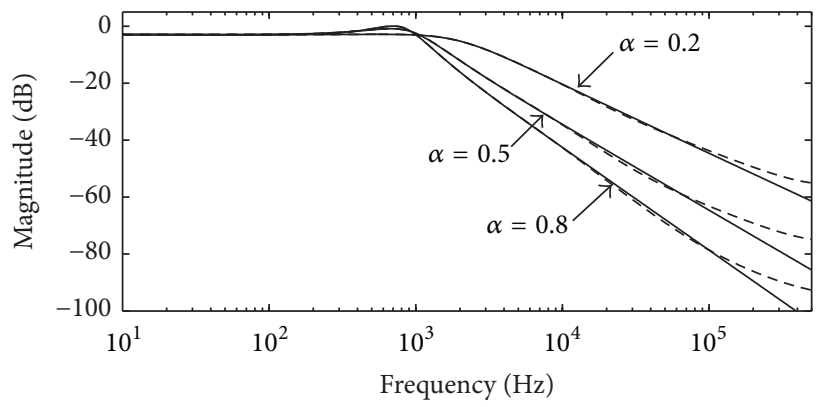

(b)

Figure 5: (a) Fractional Tow-Thomas biquad realized using approximated fractional capacitor and (b) SPICE simulated magnitude responses of $(1+\alpha)$ lowpass fractional order filter circuits for $\alpha=0.2,0.5$, and 0.8 using component values from Tables 2 and 3 .

almost 2 decades, from $200 \mathrm{~Hz}$ to $6 \mathrm{kHz}$, for the phase. In these regions, the deviation of the approximation from ideal does not exceed $1.23 \mathrm{~dB}$ and $0.23^{\circ}$ for the magnitude and phase, respectively.

Using the component values in Tables 2 and 3, the approximated fractional Tow-Thomas biquad, shown in Figure 5(a), was simulated in LTSPICE IV using LT1037 op amps to realize responses of order $(1+\alpha)=1.2,1.5$, and 1.8 . The SPICE simulated magnitude responses (dashed lines) compared to the ideal responses (solid lines) are shown in Figure 5(b).

The SPICE simulated magnitude responses show very good agreement with the MATLAB simulated ideal responses. The deviations above $20 \mathrm{kHz}$ can be attributed to the approximations of the fractional order capacitors which show significant error from their ideal behaviour above this frequency. These simulations verify that the fractional Tow-Thomas circuit can be used to realize the approximated fractional Chebyshev lowpass filter responses using approximated fractional order capacitors and that the correct selection of coefficients in the fractional order transfer function can yield ripples in the passband of the magnitude response.

\section{Conclusion}

We have proposed a new method using a nonlinear least squares optimization to determine the coefficients of fractional order transfer functions of order $(1+\alpha),(2+\alpha)$, and $(3+$ $\alpha)$ that will approximate the passband ripple characteristics of Chebyshev lowpass filters. These filter circuits were verified in simulation using approximated fractional order capacitors in a Tow-Thomas biquad circuit. This work has the potential to be applied to filters of any order and to also approximate the other traditional filter approximations using fractional order circuits that give a greater degree of control of the magnitude characteristics.

\section{Conflict of Interests}

The authors declare that there is no conflict of interests regarding the publication of this paper.

\section{References}

[1] A. S. Elwakil, "Fractional-order circuits and systems: an emerging interdisciplinary research area," IEEE Circuits and Systems Magazine, vol. 10, no. 4, pp. 40-50, 2010.

[2] A. G. Radwan, A. S. Elwakil, and A. Soliman, "Fractionalorder sinusoidal oscillators: design procedure and practical examples," IEEE Transactions on Circuits and Systems I: Regular Papers, vol. 55, no. 7, pp. 2051-2063, 2008.

[3] A. Radwan, A. Elwakil, and A. Soliman, "On the generalization of second-order filters to the fractional order domain," Journal of Circuits, Systems and Computers, vol. 18, no. 2, pp. 361-386, 2009.

[4] P. Ahmadi, B. Maundy, A. S. Elwakil, and L. Belostostski, "Highquality factor asymmetric-slope band pass filters: a fractionalorder capacitor approach," IET Circuits, Devices \& Systems, vol. 6, no. 3, pp. 187-197, 2012.

[5] A. Soltan, A. G. Radwan, and A. M. Soliman, "Fractional order filter with two fractional elements of dependant orders," Microelectronics Journal, vol. 43, no. 11, pp. 818-827, 2012.

[6] A. G. Radwan and M. E. Fouda, "Optimization of fractionalorder RLC filters," Circuits, Systems, and Signal Processing, vol. 32, no. 5, pp. 2097-2118, 2013.

[7] A. Lahiri and T. K. Rawat, "Noise analysis of single stage fractional-order low pass filter using stochastic and fractional calculus," ECTI Transactions on Electronics and Communications, vol. 7, no. 2, pp. 136-143, 2009.

[8] A. Radwan, "Stability analysis of the fractional-order $R L_{\beta} C_{\alpha}$ circuit," Journal of Fractional Calculus and Applications, vol. 3, no. 1, pp. 1-15, 2012.

[9] T. J. Freeborn, B. Maundy, and A. S. Elwakil, "Fractionalstep Tow-Thomas biquad filters," Nonlinear Theory and Its Applications, IEICE, vol. 3, no. 3, pp. 357-374, 2012. 
[10] A. S. Ali, A. G. Radwan, and A. M. Soliman, "Fractional order Butterw orth filter: active and passive realizations," IEEE Journal on Emerging and Selected Topics in Circuits and Systems, vol. 3, no. 3, pp. 346-354, 2013.

[11] M. C. Tripathy, K. Biswas, and S. Sen, "A design example of a fractional-order Kerwin-Huelsman-Newcomb biquad filter with two fractional capacitors of different order," Circuits, Systems, and Signal Processing, vol. 32, no. 4, pp. 1523-1536, 2013.

[12] M. C. Tripathy, D. Mondal, K. Biswas, and S. Sen, "Experimental studi es on realization of fractional inductors and fractionalorder bandpass filters," International Journal of Circuit Theory and Applications, 2014.

[13] A. Soltan, A. G. Radwan, and A. M. Soliman, "CCII based fractional filters of different orders," Journal of Advanced Research, vol. 5, no. 2, pp. 157-164, 2014.

[14] M. C. Tripathy, D. Mondal, K. Biswas, and S. Sen, "Design and performance study of phase-locked loop using fractionalorder loop filter," International Journal of Circuit Theory and Applications, 2014.

[15] G. Tsirimokou, C. Laoudias, and C. Psychalinos, "0.5-V fractional-order companding filters," International Journal of Circuit Theory and Applications, 2014.

[16] I. Podlubny, Fractional Differential Equations, Academic Press, San Diego, Calif, USA, 1999.

[17] M. Nakagawa and K. Sorimachi, "Basic characteristics of a fractance device," IEICE Transactions on Fundamentals of Electronics, Communications and Computer Sciences, vol. 75, pp. 1814-1819, 1992.

[18] M. Sivarama Krishna, S. Das, K. Biswas, and B. Goswami, "Fabrication of a fractional order capacitor with desired specifications: a study on process identification and characterization," IEEE Transactions on Electron Devices, vol. 58, no. 11, pp. 40674073, 2011.

[19] T. Haba, G. Ablart, T. Camps, and F. Olivie, "Influence of the electrical parameters on the input impedance of a fractal structure realised on silicon," Chaos, Solitons Fractals, vol. 24, no. 2, pp. 479-490, 2005.

[20] A. M. Elshurafa, M. N. Almadhoun, K. N. Salama, and H. N. Alshareef, "Microscale electrostatic fractional capacitors using reduced graphene oxide percolated polymer composites," Applied Physics Letters, vol. 102, no. 23, Article ID 232901, 2013.

[21] R. C. Paley and N. Wiener, Fourier Transforms in the Complex Domain, American Mathematical Society Colloquium Publication, New York, NY, USA, 19th edition, 1934.

[22] T. J. Freeborn, B. Maundy, and A. S. Elwakil, "Field programmable an alogue array implementations of fractional step filters," IET Digital Library: IET Circuits, Devices \& Systems, vol. 4, no. 6, pp. 514-524, 2010.

[23] T. Helie, "Simulation of fractional-order low-pass filters," IEEE/ACM Transactions on Audio, Speech, and Language Processing, vol. 22, no. 11, pp. 1636-1647, 2014.

[24] T. F. Coleman and Y. Li, "An interior trust region approach for nonlinear minimization subject to bounds," SIAM Journal on Optimization, vol. 6, no. 2, pp. 418-445, 1996.

[25] A. G. Radwan, A. M. Soliman, A. S. Elwakil, and A. Sedeek, "On the stability of linear systems with fractional-order elements," Chaos, Solitons \& Fractals, vol. 40, no. 5, pp. 2317-2328, 2009.

[26] I. Podlubny, I. Petráš, B. M. Vinagre, P. O’Leary, and L. Dorčák, "Analogue realizations of fractional-order controllers," Nonlinear Dynamics, vol. 29, no. 1-4, pp. 281-296, 2002.
[27] B. T. Krishna and K. V. V. S. Reddy, "Active and passive realization of fractance device of order 1/2," Active and Passive Electronic Components, vol. 2008, Article ID 369421, 5 pages, 2008. 


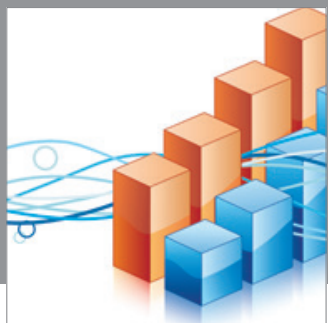

Advances in

Operations Research

mansans

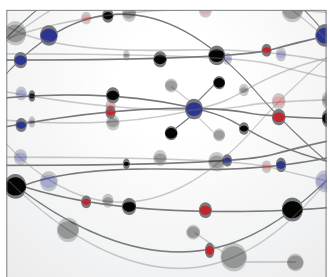

The Scientific World Journal
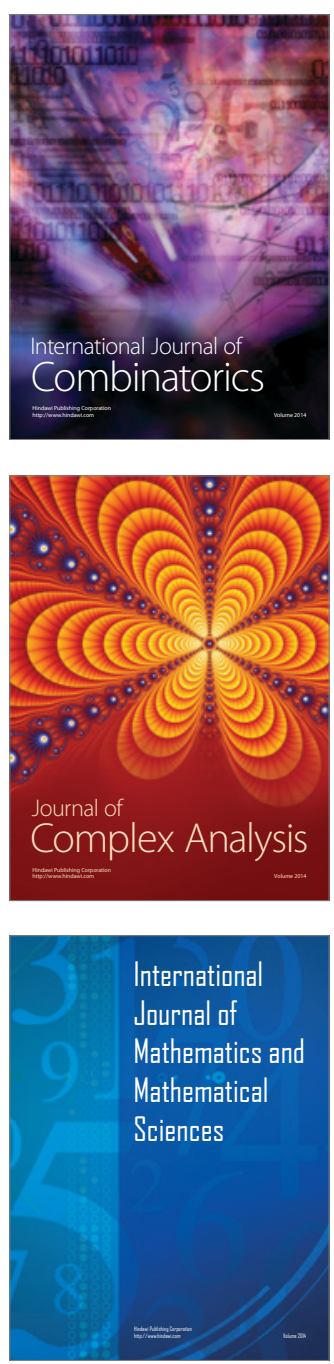
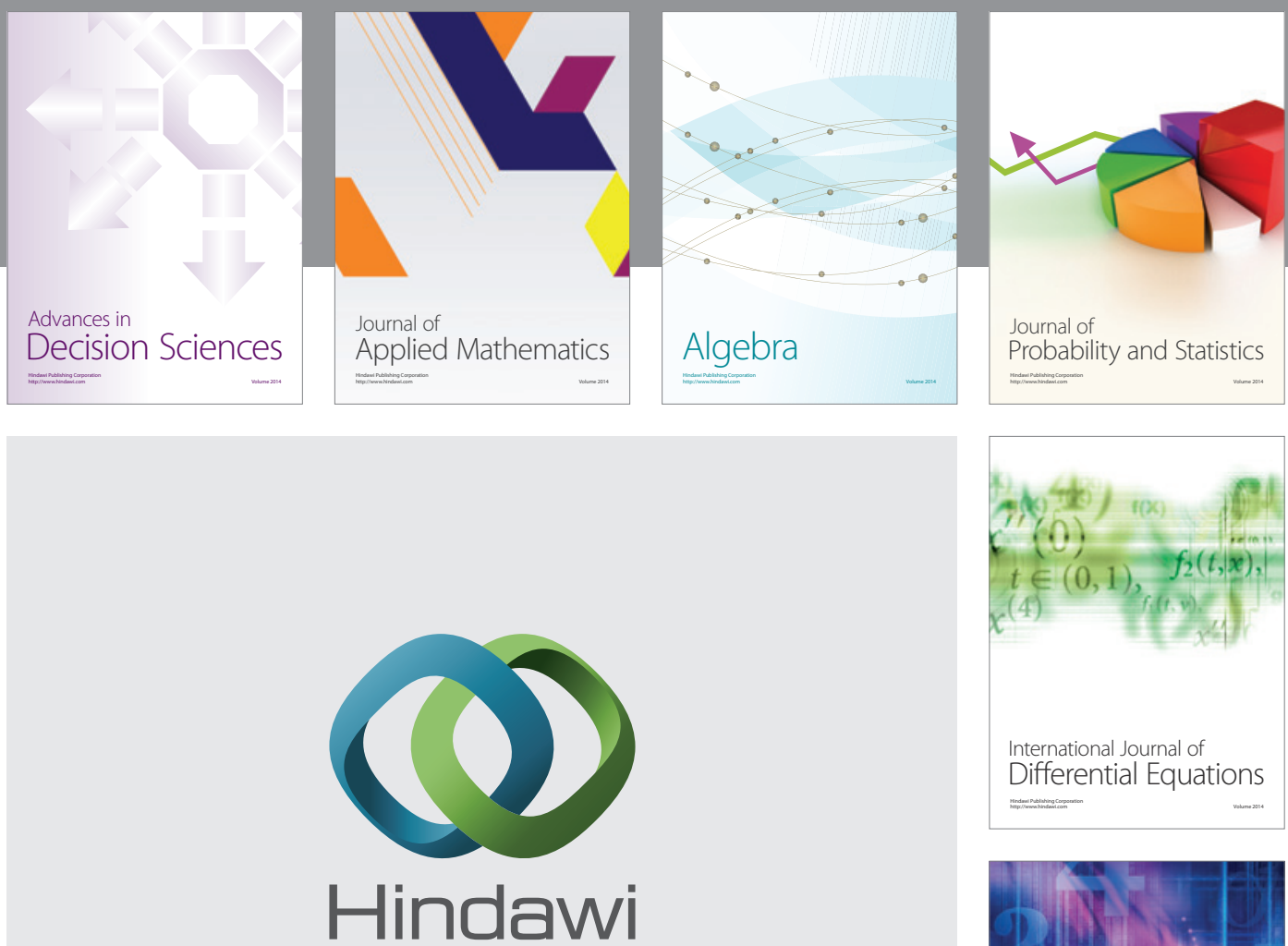

Submit your manuscripts at http://www.hindawi.com
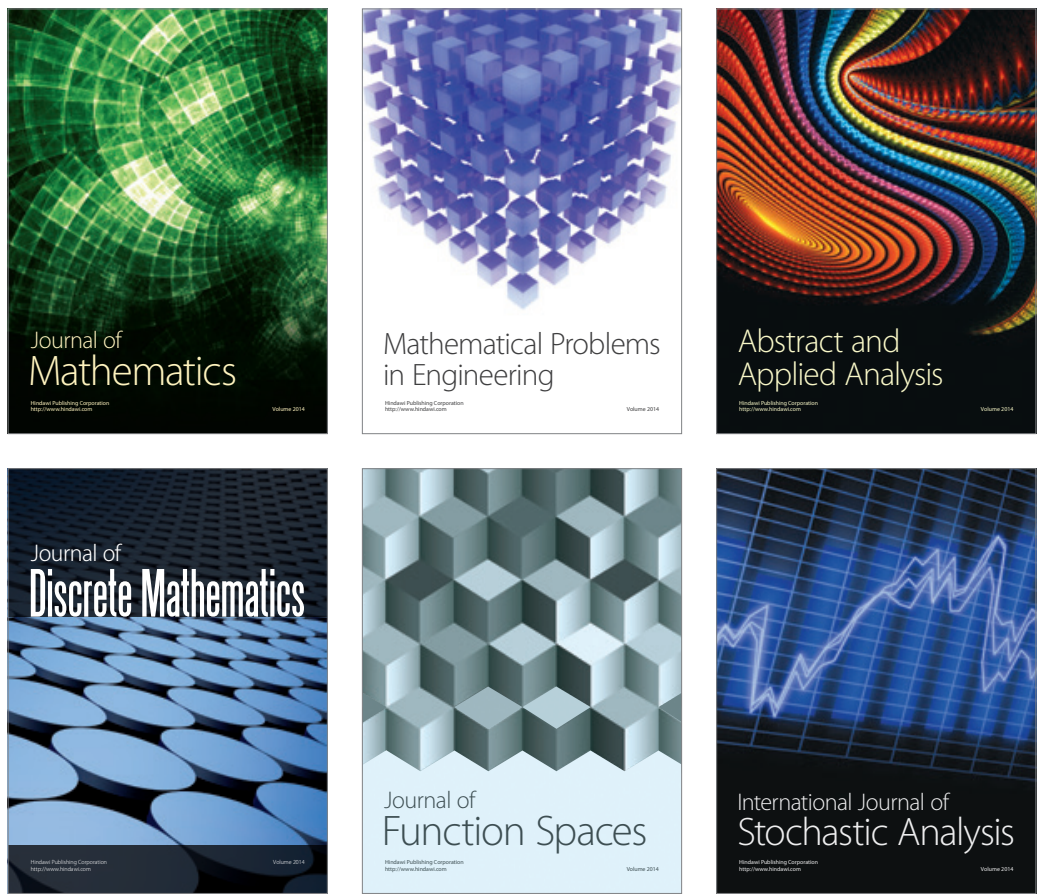

Journal of

Function Spaces

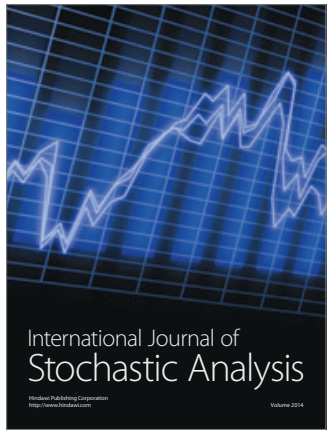

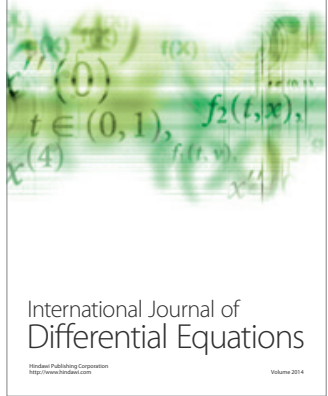
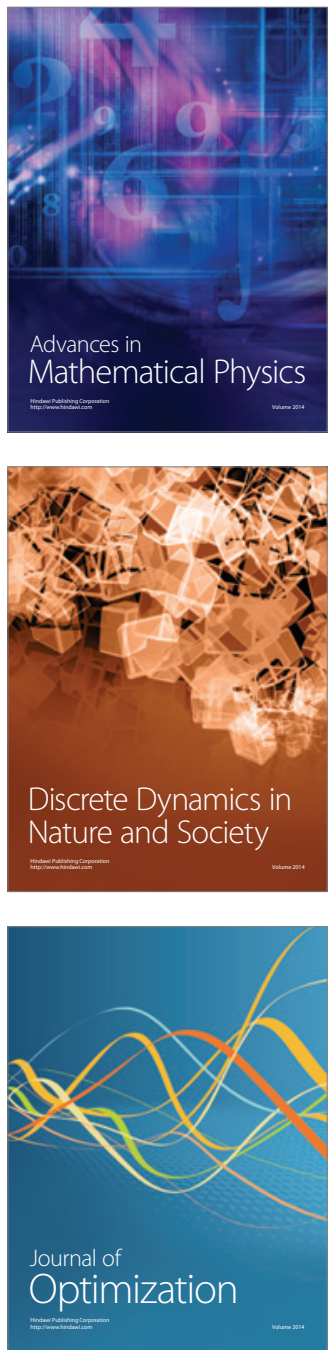